\title{
Development of novel chitosan based ketorolac implant controlled release formulation for subcutaneous drug delivery.
}

\author{
Syed Ata Ur Rahman', Sabahuddin Siddique ${ }^{1,2}$, Mohi Iqbal Mohammed Abdul ${ }^{1}$, Durdana Lateef ${ }^{3}$, \\ Shubhasis Dan ${ }^{4}$, Anirbandeep Bose ${ }^{4 *}$ \\ ${ }^{1}$ College of Pharmacy, Taibah University, Al-Madinah Al-Munawwarah, Kingdom of Saudi Arabia \\ ${ }^{2}$ Patel College of Pharmacy, Bhopal, Madhya Pradesh, India \\ ${ }^{3}$ College of Science, Taibah University, Al-Madinah Al-Munawwarah, Kingdom of Saudi Arabia \\ ${ }^{4}$ TAAB Biostudy Services, Jadavpur, Kolkata, India
}

\begin{abstract}
This present experiment deals with a formulation of novel drug delivery system of chitosan implants to modulate the release of ketorolac through the fabrication of subcutaneous implant. The implants were prepared by compression technique. The chemical stability and the characteristic features of surface morphology of the implants were confirmed by using FTIR and SEM. The thermodynamic behavior of the novel implants was investigated by using DSC/TGA. The loading capacity of Ketorolac into chitosan implants was also evaluated. The release kinetic parameters were studied in dissolution media of phosphate buffer solution (PBS). The results find that the final formulation maintains chemical stability of the drug. The morphological features of the implant confirmed uniform inclusion and distribution of ketorolac into the chitosan matrix with an evidence of porous structure. The drug release pattern of the Ketorolac exhibited controlled release behavior from chitosan implants up to 45 d governed mainly by drug diffusion mechanism. The formulated of novel implants have shown a great potential in the long term subcutaneous delivery of ketorolac.
\end{abstract}

Keywords: FTIR, SEM, Phosphate buffer solution, Drug.

Accepted on June 05, 2018

\section{Introduction}

Ketorolac can be classified as model non-steroidal antiinflammatory drugs (NSAIDs) that are highly effective for the treatment of symptomatic and non-symptomic pain and inflammation. Compare to other conventional NSAIDS ketorolac is highly effective for management of different kind of pain and inflammation [1]. The mechanism of ketorolac is mainly involved in reduction of synthesis of prostaglandins and other inflammatory chemicals that are originated from the immune system. However, oral administration of ketorolac has many shortcomings including low patient compliance [2,3]. Preparations that reduce tolerability help patients with diseases of chronic pain such as terminal cancer and lethal autoimmune disease. In a previous study, implantable drug delivery system reduced the opioid reported toxicity to a large extent [4-6]. The present experimental study aimed at designing of novel ketorolac formulation in a specially designed subcutaneous biocompatible implant that can control the drug release for more than 45 days. Thorough literature investigation of subcutaneous implants reveals that the use of the long acting biodegradable polymer chitosan to design the formulation of ketorolac has not been executed before. Therefore, our investigation to design prolonged release dosage formulation in the form of subcutaneous implant will be useful for effective treatment and management of operative and non-operative pain.

\section{Material and Methods}

Material Ketorolac hydrochloride ( $>98 \%$ HPLC) was purchased from Sigma pharmaceuticals. Chitosan, PEG 6000, Tween 80, and glycerol were also purchased from SigmaAldrich (Germany). ALL other chemicals used for analysis were of high analytical grade and purchased from Sigma Co., US 2.2.

\section{Compression technique}

Required amount of Chitosan powder, glycerol or Tween 80 PEG 6000 as mentioned in Table 1 were heated at a temperature of $75^{\circ} \mathrm{C}$ on a water bath under stirring condition with a glass rod. The exact quantity of pure Ketorolac drug was dispersed uniformly when the entire mass is in semi melted condition. The solidified blend was kept in a refrigerator for more than $1 \mathrm{~h}$. After the solidification of the mass, hard mass 
was converted to fine powder and the entire fine mass passed through a sieve (aperture $0.45 \mathrm{~mm}$ ). The fine granules were properly lubricated with talcum and compressed form $10 \mathrm{~mm}$ (flat) tablet shaped pellets. 7 different formulations were designed and formulated by using various quantity of glycerol or Tween 80 concentration [7].

Table 1. Formulations of compressed and molded implants using erosion enhancers sample formulation Ingredient (\%) Drug GMS PEG 6000 Tween 80 glycerol compressed.

\begin{tabular}{llllll}
\hline Formulation & Drug & Chitosan & PEG 6000 & Tween 80 & Glycerol \\
\hline C1 & 20 & 40 & 40 & 0 & 0.02 \\
\hline C2 & 20 & 42.5 & 37.5 & 2.5 & 0.03 \\
\hline C3 & 20 & 45 & 35 & 5 & 0.04 \\
\hline C4 & 20 & 47.5 & 32.5 & 7.5 & 0.05 \\
\hline C5 & 20 & 37.5 & 42.5 & 2.5 & 0.06 \\
\hline C6 & 20 & 35 & 45 & 0.05 & .07 \\
\hline C7 & 20 & 32.5 & 47.5 & 0.05 & 0.07 \\
\hline
\end{tabular}

\section{Chemical integrity of the prepared implants FT-IR spectra}

The compressed implant in the form of tablets were crushed into powder form and the chemical stability of the powder samples were analyzed by a using a Perkin Elmer Spectrum 2000 FT-IR spectrometer, universal FTIR spectrum series at a resolution of $4 \mathrm{~cm}^{-1}[8]$.

\section{Thermodynamic behavior of the ketorolac-loaded implants}

The thermal behavior of the compressed implants in the form of tablets was evaluated by using Differential Scanning Calorimeter (DSC) and thermal gravimetric analysis (TGA). The required quantity of the samples $(100 \pm 1 \mathrm{mg})$ was placed in platinum crucible with a specified heating range of $25-500^{\circ} \mathrm{C}$ with a heating rate of $10 \mathrm{C} / \mathrm{min}$ [9].

\section{Morphological features of the ketorolac-loaded implants}

The internal morphological features and surface area of the ketorolac loaded implants was investigated by using Scanning Electron Microscope (SEM). Samples were undergone the process of gold-sputter coating (SPI Module ${ }^{\mathrm{TM}}$ Sputter Coater, SPI Supplies, PA) for the purpose of rendering electrically conductivity to the samples before analysis [10].

\section{In vitro drug release}

Actual concentration of loaded ketorolac in prepared implants was estimated by grinding ketorolac-loaded implants and extracting ketorolac in DMSO by at a temperature of $40^{\circ} \mathrm{C}$ for more than $6 \mathrm{~h}$. The final solution was then properly filtered and diluted with Phosphate buffer solution. The concentration of ketorolac was then estimated UV spectrophotometry. All measurements were performed in triplicate and the encapsulation efficiency and the percentage of loaded ketorolac was calculated. The in vitro drug release of ketorolac implant was calculated in $\mathrm{PBS}\left(\mathrm{pH} 7.4 ; 37^{\circ} \mathrm{C}\right)$ at different time intervals using UV-vis spectrophotometer (Lambda $25 \mathrm{UV} / \mathrm{Vis}$ Spectrophotometer, PerkinElmer, MA, USA) at a wavelength of $271 \mathrm{~nm}$.

\section{Mathematical modeling of release kinetics}

In order to evaluate the release mechanism of the ketorolac the percentage of drug release data were compared with the fundamental mathematical models of zero order, diffusion and using the Korsmeyer-Peppas equation.

\section{Results}

\section{FT-IR spectrum of chitosan implants}

Chemical integrity of the prepared implants evaluated by the FT-IR spectra of ketorolac-loaded implants are illustrated in Figure 1. A strong band in the region $3611 \mathrm{~cm}^{-1}$ corresponds to $\mathrm{N}-\mathrm{H}$ and $\mathrm{O}-\mathrm{H}$ stretching, as well as the intramolecular hydrogen bonds. The absorption bands at around $2921 \mathrm{~cm}^{-1}$ can be attributed to C-H symmetric and asymmetric stretching, respectively. These bands are characteristics of typical polysaccharides. First, the characteristic bands of the basic polymers were observed as follows; the main characteristic bands of chitosan were observed at $1652 \mathrm{~cm}^{-1}$ corresponding to carbonyl stretching, bands at 1423 and $1112 \mathrm{~cm}^{-1}$ indicating $\mathrm{CAC}$ and CAO stretching in chitosan.

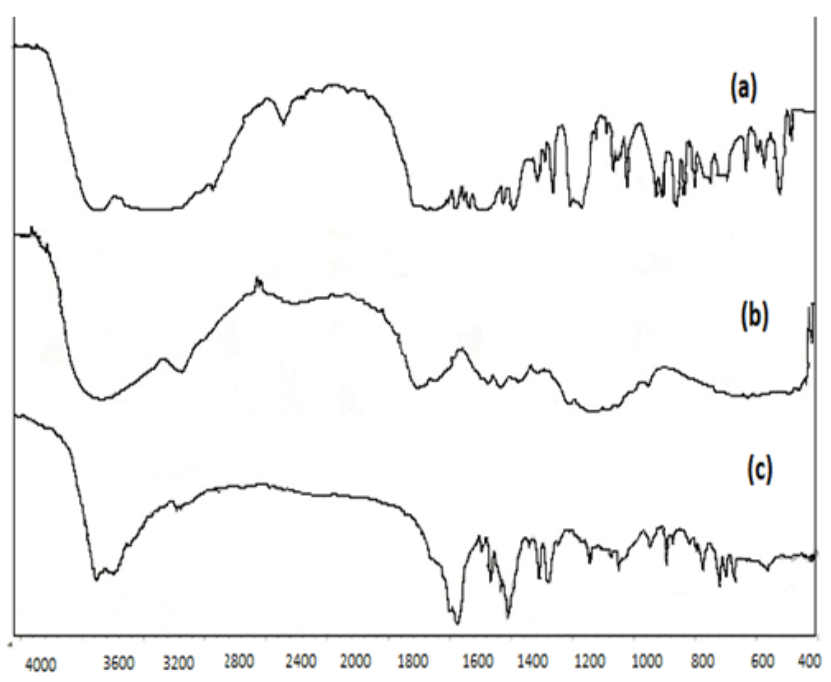

Figure 1. FTIR spectrum of chitosan implants.

Ketorolac showed major characteristic bands as follows (3348 $\mathrm{cm}^{-1}$ (NH stretch), $1726 \mathrm{~cm}^{-1}(\mathrm{C}=\mathrm{O}$ the vibration stretching of carboxylic acid groups stretch for acid) and $1172 \mathrm{~cm}^{-1} \mathrm{C}=\mathrm{O}$ for diaryl ketone). In Figure 1, the FTIR spectrum of final chitosan-implant representing the chemical integrity of the ketorolac loaded in chitosan implants. It is clearly evident that the entire major characteristic peak which corresponds to 
identify of both chitosan and ketorolac are clearly visible. So the chemical integrity and stability of the drug and polymer in the final form of chitosan implant are intact.

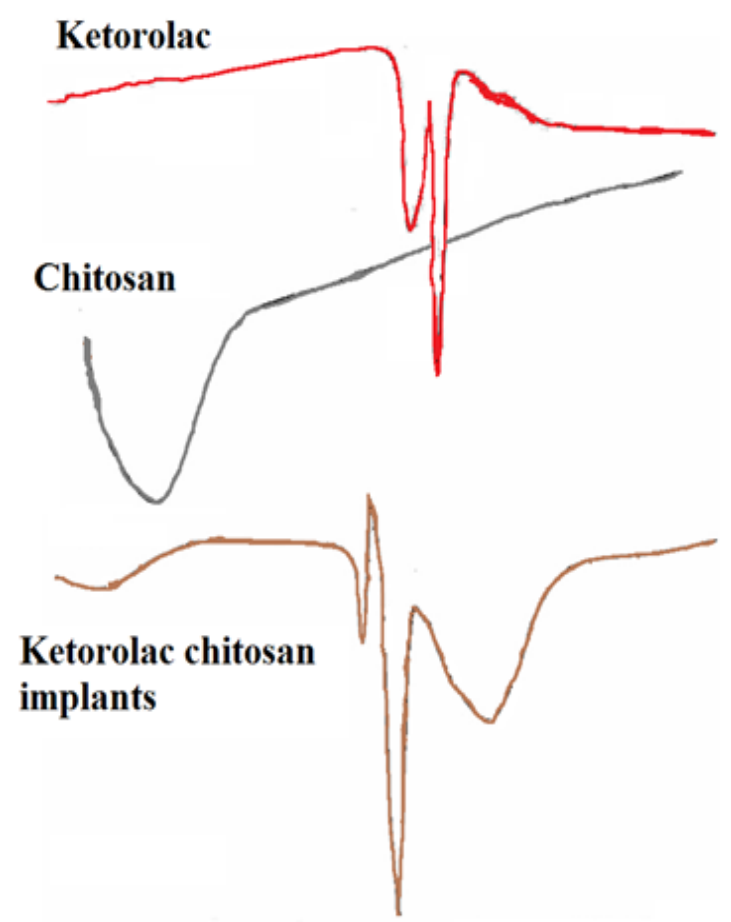

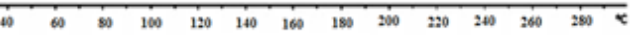

Figure 2. Characteristic features of thermal behavior of Chitosan implants.

\section{Thermodynamic behavior of the ketorolac-loaded implants}

The thermal behavior of ketorolac-loaded implants was determined by DSC as shown in Figure 2. The native carbohydrate polymer; chitosan showed one characteristic peak at $60^{\circ} \mathrm{C}$ corresponding to $\mathrm{Tm}$ of chitosan as early reported. Ketorolac exhibited a long and sharp characteristic endothermic peak at $163.04^{\circ} \mathrm{C}$ due to its phase transition system. The powder grinded from chitosan implant of Ketorolac shows characteristic peak at $158.90^{\circ} \mathrm{C}$. It showed the slight change in characteristic peak may be due to fusion of excipient present in the physical mixture. From this result, it clears that there is no interaction in between Ketorolac and excipients.

\section{Morphology of the ketorolac-loaded implants}

The ketorolac-loaded implants morphology and ketorolac-free implants were investigated using SEM. Ketorolac-free implants exhibited amorphous smooth surfaces (Chitosan) (Figures 3 and 4), while ketorolac-loaded implants showed a porous structure based morphology and rough surfaces. The characteristics features of this implant were due to with increased concentrations of ketorolac embedded in the implant.

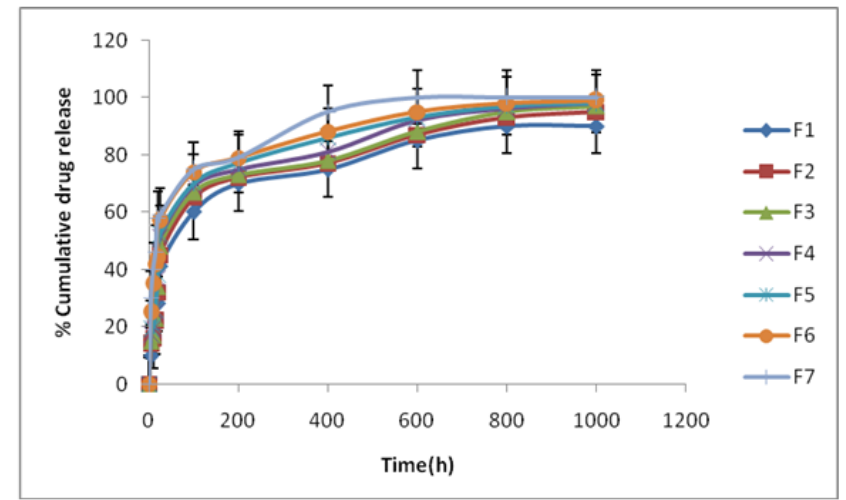

Figure 3. Percentage cumulative drug release of various formulations at different time interval.

\section{Drug loading efficiency and in vitro drug release}

The entrapment efficiency values ranged from $98.00 \pm 0.70 \%$ for all the formulation. The cumulative drug release $(\% \mathrm{CDR})$ profiles of ketorolac-loaded implants are presented in Figure 4. Ketorolac exhibited controlled release behavior from chitosan implants up to $1000 \mathrm{~h}$. The formulation F5 shows a longer duration of time.

\section{In vitro drug release kinetics}

In vitro ketorolac release from ketorolac-loaded implants was fitted using different mathematical models and the kinetic parameters are listed in Table 2. It is evident from the release figure that the plots are curvilinear suggesting that the release process is not zero-order in nature. The release pattern is very close to Korsmeyer-peppas model.

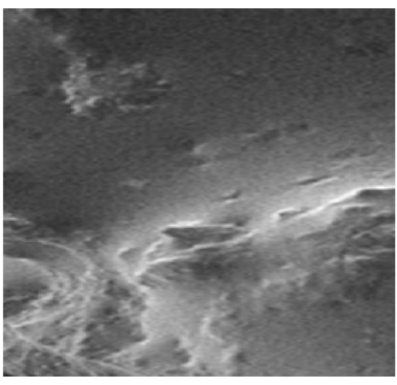

(a)

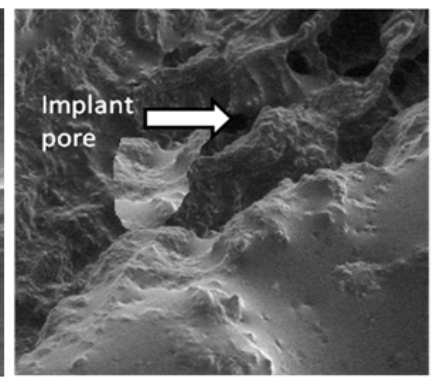

(b)
Figure 4. Scanning electron microscopy of: (a) Chitosan powder; (b) Chitosan drug implants.

Table 2. Drug release kinetics parameters based on various dissolution models.

\begin{tabular}{lllll}
\hline \multirow{2}{*}{$\begin{array}{l}\text { Formula } \\
\text { code }\end{array}$} & \multicolumn{2}{l}{$\mathbf{R}^{2}$-value } & & \\
\cline { 2 - 5 } & Zero-order & Diffusion & Korsmeyer-peppas model & n value \\
\hline F1 & 0.722 & 0.912 & 0.934 & 0.343 \\
\hline F2 & 0.745 & 0.923 & 0.945 & 0.359 \\
\hline F3 & 0.789 & 0.912 & 0.958 & 0.365 \\
\hline
\end{tabular}




\begin{tabular}{lllll}
\hline F4 & 0.734 & 0.936 & 0.968 & 0.358 \\
\hline F5 & 0.698 & 0.915 & 0.959 & 0.547 \\
\hline F6 & 0.758 & 0.936 & 0.965 & 0.525 \\
\hline F7 & 0.739 & 0.915 & 0.935 & 0.565 \\
\hline
\end{tabular}

\section{Discussion}

Subcutaneous chitosan implant is formulated and designed by many researchers. Most of the work of subcutaneous drug delivery where chitosan and other polymers were used is based on longer duration of release profile [7,11-13]. Use of various polymers other than chitosan also has been investigated by various researchers but the results are not found very satisfactory. FTIR investigation is indicating that the interaction between physical and chemical aspect is minimal and can be neglected. In respect of melting point ketorolac in implant have a minimum shift as compare to raw drugs. Minimal shifting of melting point indicates the thermal behavior of the polymers and the raw drug has been not changed which is a positive aspect of chitosan implants.

The ketrolac drug release pattern an important criterion for successful development of subcutaneous implant drug delivery system. The dissolution study clearly indicating that chitosan based implant formulation are highly successful for delivering the drug release up to 45 days. For successful management of terminal cancer pain long-term medication in the form of subcutaneous implant dosage form may be an effective alternative of opoid based analgesic. Scanning electron microscopy of the final formulation is showing smaller pores like structure is present in the implant formulation which definitely creates a sustained property of the chitosan implant. In addition the release kinetic behavior is following Korsmear model which is showing the implant possess good characteristics of sustained release formulation. Most of the formulation is showing good release pattern among them F1 formulation is sustaining the release pattern for longer hours. As the $n$ value of all the formulation is below 0.5 . So it is clearly evident that the release pattern follows quasi Fickian diffusion. Therefore, the final results of the kinetic study permit us to make a conclusion that the chitosan based fabricated implant are able to prolong the drug release through diffusion pore for longer duration of time.

\section{Conclusion}

CHITOSAN polymer represented a good candidate for implant, where obtained integrity was intact and ketorolac release was sustained for more than $700 \mathrm{~h}$. Moreover, the presented complex did not affect the chemical integrity or stability of the drug. The polymer proved its biodegradation and weight loss during the experimentation period. The in vitro study suggested that the proposed system can be valid for sustaining the drug for at least $45 \mathrm{~d}$ in vivo. Ketorolac exhibited controlled release behavior from Chitosan implants up to 1080 h.

\section{Acknowledgment}

The present study was supported by Acharya and BM Reddy college of Pharmacy, Bangalore-560107.

References

1. Kuritzky L, Samraj GP. Nonsteroidal anti-inflammatory drugs in the treatment of low back pain. J Pain Res 2012; 5: 579-590.

2. Modi CM, Mody SK, Patel HB, Dudhatra GB, Kumar A, Avale M. Toxicopathological overview of analgesic and anti-inflammatory drugs in animals. J Appl Pharm Sci 2012; 2: 149-157.

3. Rogers E, Mehta S, Shengelia R, Reid MC. Four strategies for managing opioid-induced side effects in older adults. Clin Geriatr 2013; 21.

4. Smith TJ, Staats, PS, Deer T, Stearns LJ, Rauck RL, Boortz-Marx RL, Buchser E, Català E, Bryce DA, Coyne PJ, Pool GE. Implantable Drug delivery systems study group. Randomized clinical trial of an implantable drug delivery system compared with comprehensive medical management for refractory cancer pain: impact on pain, drug-related toxicity, and survival. J Clin Oncol 2002; 20: 4040-4049.

5. Mabrouk, Mostafa H, Beherei, Hanan, Elshebiney, Shaimaa, Tanaka, Masaru. Newly developed controlled release subcutaneous formulation for tramadol hydrochloride. Saudi Pharm J 2018; 26.

6. Thiresen G, Yahya EC, Pradeep K, Divya B, Lisa du TC, Girish M, Dinesh N, Viness P. Implantable and transdermal polymeric drug delivery technologies for the treatment of central nervous system disorders. Pharm Development Technol 2016; 22: 476-486.

7. Mathur VB, Mudnik R, Roy A, Shivhare U, Bhusari K. Formulation and evaluation of controlled release antibiotic biodegradable implants for post-operative site delivery. Acta Pharm 2010; 60: 111-117.

8. Canh Le T, Monique L, Pompilia I-S, Mircea AM. Nacylated chitosan: hydrophobic matrices for controlled drug release. J Controlled Release 2003; 93: 1-13.

9. Alves R, Reis TVS, Silva LCC, Storpírtis S, Mercuri LP, Matos JR. Thermal behavior and decomposition kinetics of rifampicin polymorphs under isothermal and nonisothermal conditions Brazilian. J Pharm Sci 2010; 46: 343-351.

10. Mangano F, Raspanti M, Maghaireh H, Mangano C. Scanning electron microscope (SEM) evaluation of the interface between a nanostructured calcium-incorporated dental implant surface and the human bone. Materials 2017; 10: 1438.

11. Uddin N, Uddin M, Kabir T. Simultaneous estimation of ketorolac tromethamine and tiemonium methylsulphate in biofluids by a validated UPLC method. Indo J Pharm Sci Tech 2008; 5: 15-25.

12. Yi-Chia L, Kuo-Sheng L, Demei L, Min-Jhan L, ShihJung L, Hiroshi I. In vivo and in vitro elution of analgesics from multi-layered Poly (D, L)-lactide-co- 
Development of novel chitosan based ketorolac implant controlled release formulation for subcutaneous drug delivery

glycolide nanofibers incorporated ureteral stents. J Nanomaterials 2018.

13. Brito MK, Schellini SA, Padovani CR, Pellizzon CH, Trindade-Neto CG. Chitosan inclusions in the subcutaneous space of rats: clinic, histologic and morphometric evaluation. An Bras Dermatol 2009; 84: $35-40$.

\section{*Correspondence to}

Anirbandeep Bose

TAAB Biostudy Services

Jadavpur

Kolkata

India 